\title{
THE DIRAC OPERATOR ON COMPACT QUANTUM GROUPS
}

\author{
SERGEY NESHVEYEV AND LARS TUSET
}

Dedicated to the memory of Gerard J. Murphy

\begin{abstract}
For the $q$-deformation $G_{q}, 0<q<1$, of any simply connected simple compact Lie group $G$ we construct an equivariant spectral triple which is an isospectral deformation of that defined by the Dirac operator $D$ on $G$. Our quantum Dirac operator $D_{q}$ is a unitary twist of $D$ considered as an element of $U \mathfrak{g} \otimes \mathrm{Cl}(\mathfrak{g})$. The commutator of $D_{q}$ with a regular function on $G_{q}$ consists of two parts. One is a twist of a classical commutator and so is automatically bounded. The second is expressed in terms of the commutator of the associator with an extension of $D$. We show that in the case of the Drinfeld associator the latter commutator is also bounded.
\end{abstract}

\section{INTRODUCTION}

The Dirac operator on Minkowski space was introduced in 1928 by P. Dirac who sought a first order differential operator with square equal to the Laplacian and found in effect the fundamental mechanisms governing spin-half particles obeying Fermi statistics. Its generalization by Atiyah and Singer plays an essential role in index theory, mathematical physics and representation theory, and its axiomatization in terms of spectral triples is at the heart of Connes' non-commutative geometry [7].

Quantum groups being quantizations of Poisson Lie groups should by all accounts be noncommutative manifolds, but it has proved difficult to put them rigorously into Connes' framework. In the quest for an appropriate Dirac operator on quantum groups and their homogeneous spaces basically two approaches have been adopted.

One consists of developing $q$-analogues of standard differential geometric notions, but this poses several immediate problems. Firstly, it is not clear what a quantum Clifford algebra should be. The natural suggestion using braidings [2, 23, 25] seems reasonable only when the braiding is sufficiently simple, e.g. a Hecke symmetry, see for instance the discussion in [23. Secondly, differential calculi are defined in terms of elements of quantized universal enveloping algebras that do not act as derivations and yield unbounded commutators with some regular functions, see e.g. [35], rendering this approach successful so far only for irreducible quantum flag manifolds. The case of the quantum 2-sphere is considered in [34, 13, 36, 32] and the general case is due to Krähmer [31], who circumvents the Clifford algebra problem at the cost of including operators with unconventional classical limits, see the remark after [31, Prop. 2]. Also, spectral triples obtained using this approach cannot be expected to be regular [33].

The other approach was suggested by Connes and Landi [9] and consists of looking for isospectral deformations of Dirac operators. To write down explicitly what this means and to handle such operators efficiently requires a good understanding of Clebsch-Gordan coefficients and spectral properties of classical Dirac operators. The first result in this direction was obtained by Chakraborty and Pal [3], who constructed a spectral triple on $\mathrm{SU}_{q}(2)$ which was then studied in detail by Connes [8]. Their Dirac operator although not exactly an isospectral deformation of the classical one, is closely related [4] to such an operator proposed meanwhile by Da̧browski et al [12]. Similar results are obtained for 2- and 4-dimensional quantum spheres [10, 11]. Finally, a class of operators on quantum

Date: March 21, 2007.

Supported by the Research Council of Norway. 
$\mathrm{SU}(N)$ and the odd-dimensional spheres is introduced in [5], but again it seems difficult to single out which of these operators have the right classical limit.

The two approaches should be related in the same way as the universal $R$-matrix is related to the element $t \in \mathfrak{g} \otimes \mathfrak{g}$ defined by the symmetric invariant form; although we are not going to discuss this issue in this paper, see Example 2.6 below.

Our construction of the Dirac operator on the $q$-deformation $G_{q}$ of a group $G$ is inspired by work of Fiore [22, 24], brought to our attention by Ulrich Krähmer. Let $V$ be a finite dimensional $\mathfrak{g}$-module with fixed invariant symmetric form. The Clifford algebra $\mathrm{Cl}(V)$ is semi-simple and thus has no nontrivial deformations. For a fixed algebra isomorphism $\varphi: U_{h} \mathfrak{g} \rightarrow U \mathfrak{g}[[h]]$, Fiore seeks a map $V[[h]] \rightarrow \mathrm{Cl}(V)[[h]]$ which coincides with the embedding $V \rightarrow \mathrm{Cl}(V)$ modulo $h$ and is $U_{h} \mathfrak{g}-$ equivariant. He shows that such a map can be gotten by using a twist, i.e. an element $\mathcal{F} \in$ $(U \mathfrak{g} \otimes U \mathfrak{g})[[h]]$ such that $(\varphi \otimes \varphi) \hat{\Delta}_{h}=\mathcal{F} \hat{\Delta} \varphi(\cdot) \mathcal{F}^{-1}$.

Consider now the Dirac operator on $G$ regarded as an element $\mathcal{D}$ of $U \mathfrak{g} \otimes \mathrm{Cl}(\mathfrak{g})$. In the formal deformation setting define the quantum Dirac operator as the element of $U_{h} \mathfrak{g} \otimes \mathrm{Cl}(\mathfrak{g})$ obtained by conjugating $\left(\varphi^{-1} \otimes \iota\right)(\mathcal{D})$ by $\left(\varphi^{-1} \otimes \widetilde{\mathrm{ad}}\right)(\mathcal{F})$, where $\widetilde{\mathrm{ad}}: U \mathfrak{g} \rightarrow \mathrm{Cl}(\mathfrak{g})$ is induced by the Lie algebra homomorphism $\mathfrak{g} \rightarrow \mathfrak{s o}(\mathfrak{g})$. This can also be done for real parameters $h=2 \log q$, but then instead of $U_{q} \mathfrak{g}$ and $U \mathfrak{g}$ one has to consider appropriate completions.

Although twists exist, their analytical properties are difficult to study. From a representation theoretical point of view, however, it is not the twist but the associator and braiding that matter. More precisely, by a famous result of Drinfeld [14, 15] the appropriate braided tensor category of $U_{h} \mathfrak{g}-$ modules with braiding defined by the universal $R$-matrix and with trivial associativity morphisms is equivalent to a category of $U \mathfrak{g}[[h]]$-modules with braiding given by $e^{h t / 2}$ and associativity constraints defined by the monodromy of the Knizhnik-Zamolodchikov (KZ) equations. Then choosing a twist essentially means that one fixes such an equivalence of categories. In the same spirit we show that analytic properties of our quantum Dirac operator are determined by the associator rather than the twist, and indeed a form of the KZ-equations is used crucially to show that we actually get a spectral triple.

\section{DRINFELD ASSOCIATOR}

Let $G$ be a simply connected simple compact Lie group, $\mathfrak{g}$ its complexified Lie algebra. Fix a maximal torus in $G$, and let $\mathfrak{h} \subset \mathfrak{g}$ be the corresponding Cartan subalgebra. Choose a system $\left\{\alpha_{1}, \ldots, \alpha_{n}\right\}$ of simple roots. Let $\left(a_{i j}\right)_{1 \leq i, j \leq n}$ be the Cartan matrix of $\mathfrak{g}$, and $d_{1}, \ldots, d_{n}$ be coprime positive integers such that $\left(d_{i} a_{i j}\right)_{i, j}$ is symmetric. Define as usual a bilinear form on $\mathfrak{h}^{*}$ by $\left(\alpha_{i}, \alpha_{j}\right)=$ $d_{i} a_{i j}$. For each integral dominant weight $\lambda$ we fix an irreducible unitary representation $\pi_{\lambda}: G \rightarrow$ $B\left(V_{\lambda}\right)$ with highest weight $\lambda$. Then the group von Neumann algebra $W^{*}(G)$ of $G$ is the $\mathrm{C}^{*}$-product of the algebras $B\left(V_{\lambda}\right)$. The algebra $\mathcal{U}(G)$ of unbounded operators affiliated with $W^{*}(G)$ is the algebraic product $\prod_{\lambda} B\left(V_{\lambda}\right)$. We denote by $\hat{\Delta}$ the comultiplication $W^{*}(G) \rightarrow W^{*}(G) \bar{\otimes} W^{*}(G)$. It extends to a $*$-homomorphism $\mathcal{U}(G) \rightarrow \mathcal{U}(G \times G)=\prod_{\lambda, \mu} B\left(V_{\lambda} \otimes V_{\mu}\right)$ which we denote by the same symbol.

For $q \in(0,1)$ denote by $G_{q}$ the $q$-deformation of $G$. To fix notation, recall that the algebra $\mathcal{U}\left(G_{q}\right)$ of unbounded operators affiliated with the von Neumann algebra $W^{*}\left(G_{q}\right)$ contains the algebra $U_{q} \mathfrak{g}$ generated by $X_{i}, Y_{i}, K_{i}, K_{i}^{-1}(1 \leq i \leq n)$ such that the relations

$$
\begin{gathered}
K_{i} K_{i}^{-1}=K_{i}^{-1} K_{i}=1, \quad K_{i} K_{j}=K_{j} K_{i}, \quad K_{i} X_{j} K_{i}^{-1}=q_{i}^{a_{i j} / 2} X_{j}, \quad K_{i} Y_{j} K_{i}^{-1}=q_{i}^{-a_{i j} / 2} Y_{j}, \\
X_{i} Y_{j}-Y_{j} X_{i}=\delta_{i j} \frac{K_{i}^{2}-K_{i}^{-2}}{q_{i}-q_{i}^{-1}}
\end{gathered}
$$


as well as the quantum Serre relations are satisfied, where $q_{i}=q^{d_{i}}$. The algebra $U_{q} \mathfrak{g}$ is a Hopf *-algebra with comultiplication $\hat{\Delta}_{q}$ and involution given by

$$
\begin{gathered}
\hat{\Delta}_{q}\left(K_{i}\right)=K_{i} \otimes K_{i}, \quad \hat{\Delta}_{q}\left(X_{i}\right)=X_{i} \otimes K_{i}+K_{i}^{-1} \otimes X_{i}, \quad \hat{\Delta}_{q}\left(Y_{i}\right)=Y_{i} \otimes K_{i}+K_{i}^{-1} \otimes Y_{i}, \\
K_{i}^{*}=K_{i}, \quad X_{i}^{*}=Y_{i} .
\end{gathered}
$$

Denote by $\mathcal{R} \in \mathcal{U}\left(G_{q} \times G_{q}\right)$ the universal $R$-matrix, see e.g. [6, Theorem 8.3.9] for an explicit formula. It is the unique element satisfying the following two properties. We have

$$
\hat{\Delta}_{q}^{o p}=\mathcal{R} \hat{\Delta}_{q}(\cdot) \mathcal{R}^{-1}
$$

and if $\pi_{\lambda, q}$ is a finite dimensional representation with a highest weight vector $\xi_{\lambda}$ (that is, $\pi_{\lambda, q}\left(X_{i}\right) \xi_{\lambda}=$ 0 and $\pi_{\lambda, q}\left(K_{i}\right) \xi_{\lambda}=q_{i}^{\lambda\left(H_{i}\right) / 2} \xi_{\lambda}$, where $H_{i} \in \mathfrak{h}$ is such that $\left.\alpha_{j}\left(H_{i}\right)=a_{i j}\right)$ and $\pi_{\mu, q}^{\prime}$ a finite dimensional representation with a lowest weight vector $\xi_{\mu}^{\prime}\left(\right.$ so $\pi_{\mu, q}^{\prime}\left(Y_{i}\right) \xi_{\mu}^{\prime}=0$ and $\left.\pi_{\mu, q}^{\prime}\left(K_{i}\right) \xi_{\mu}^{\prime}=q_{i}^{\mu\left(H_{i}\right) / 2} \xi_{\mu}^{\prime}\right)$ then

$$
\left(\pi_{\lambda, q} \otimes \pi_{\mu, q}^{\prime}\right)(\mathcal{R})\left(\xi_{\lambda} \otimes \xi_{\mu}^{\prime}\right)=q^{(\lambda, \mu)} \xi_{\lambda} \otimes \xi_{\mu}^{\prime} .
$$

Since finite dimensional representations of $G_{q}$ are again classified by integral dominant weights, we have a canonical identification of the centers of $W^{*}\left(G_{q}\right)$ and $W^{*}(G)$. It extends to a *-isomorphism $W^{*}\left(G_{q}\right) \cong W^{*}(G)$, and therefore $\mathcal{U}\left(G_{q}\right) \cong \mathcal{U}(G)$. Such an isomorphism does not respect comultiplications, and to compare them we recall the notion of the Drinfeld associator, see e.g. [17] and [26] for more details.

Let $A$ and $B$ be operators on a finite dimensional vector space $V$. Put

$$
\hbar=\frac{\log q}{\pi i} .
$$

Consider the differential equation

$$
G^{\prime}(x)=\hbar\left(\frac{A}{x}+\frac{B}{x-1}\right) G(x)
$$

where $G:(0,1) \rightarrow \operatorname{End}(V)$. Assume that neither $A$ nor $B$ has eigenvalues which differ by a nonzero integral multiple of $\frac{1}{\hbar}$. Then there exist unique solutions $G_{0}$ and $G_{1}$ such that the functions $G_{0}(x) x^{-\hbar A}$ and $G_{1}(1-x) x^{-\hbar B}$ extend to holomorphic functions in the unit disc with value 1 at $x=0$. These solutions are in fact $\mathrm{GL}(V)$-valued, hence there exists $\Phi(A, B) \in \mathrm{GL}(V)$ such that

$$
G_{0}(x)=G_{1}(x) \Phi(A, B) \text { for all } x \in(0,1) .
$$

We will be interested only in the case when $V$ is a Hilbert space and the operators $A$ and $B$ are self-adjoint. Then the assumptions on the spectra are automatically satisfied. For $a \in(0,1)$ let $G_{a}$ be the unique solution such that $G_{a}(a)=1$. Note that $G_{a}(x)$ is unitary. Indeed, $\hbar\left(\frac{A}{x}+\frac{B}{x-1}\right)$ is skew-adjoint, so $G_{a}$ is an integral curve of a time-dependent vector field on the unitary group. By uniqueness of solutions we have $G_{a}(x)=G_{0}(x) G_{0}(a)^{-1}$, so

$$
a^{-\hbar B} G_{a}(1-a) a^{\hbar A}=a^{-\hbar B} G_{0}(1-a) G_{0}(a)^{-1} a^{\hbar A}=a^{-\hbar B} G_{1}(1-a) \Phi(A, B) G_{0}(a)^{-1} a^{\hbar A} .
$$

Since $a^{-\hbar B}$ is unitary for any $a \in(0,1)$, the operators

$$
a^{-\hbar B} G_{1}(1-a)=a^{-\hbar B}\left(G_{1}(1-a) a^{-\hbar B}\right) a^{\hbar B}
$$

converge to 1 as $a \rightarrow 0^{+}$. Similarly $G_{0}(a)^{-1} a^{\hbar A} \rightarrow 1$ as $a \rightarrow 0^{+}$. It follows that

$$
\Phi(A, B)=\lim _{a \rightarrow 0^{+}} a^{-\hbar B} G_{a}(1-a) a^{\hbar A} .
$$

This expression makes it in particular obvious that $\Phi(A, B)$ is unitary. 
Consider the rescaling $(\cdot, \cdot)$ of the Killing form on $\mathfrak{g}$ such that its restriction to $\mathfrak{h}$ is the one induced by the symmetric form on $\mathfrak{h}^{*}$ defined above. Let $\left\{x_{k}\right\}_{k}$ be a basis in the real Lie algebra of $G$ such that $\left(x_{k}, x_{l}\right)=-\delta_{k l}$. Put

$$
t=-\sum_{k} x_{k} \otimes x_{k} \in \mathfrak{g} \otimes \mathfrak{g} \subset \mathcal{U}(G \times G) .
$$

This element is self-adjoint, e.g. because $x_{k}$ lie in the real Lie algebra of $G$ and so $x_{k}^{*}=-x_{k}$. The Drinfeld associator is defined by

$$
\Phi_{K Z}=\Phi\left(t_{12}, t_{23}\right) .
$$

More precisely, it is the unique unitary element in $W^{*}(G) \bar{\otimes} W^{*}(G) \bar{\otimes} W^{*}(G)$ such that for any finite dimensional representations $\pi_{i}: G \rightarrow B\left(V_{i}\right), i=1,2,3$, we have

$$
\left(\pi_{1} \otimes \pi_{2} \otimes \pi_{3}\right)\left(\Phi_{K Z}\right)=\Phi\left(\left(\pi_{1} \otimes \pi_{2} \otimes \pi_{3}\right)(t \otimes 1),\left(\pi_{1} \otimes \pi_{2} \otimes \pi_{3}\right)(1 \otimes t)\right) .
$$

The following variant of a famous result of Drinfeld will play a central role in the paper.

Theorem 1.1. There exist a *-isomorphism $\varphi: W^{*}\left(G_{q}\right) \rightarrow W^{*}(G)$ extending the canonical identification of the centers and a unitary $\mathcal{F} \in W^{*}(G) \bar{\otimes} W^{*}(G)$ such that

(i) $(\varphi \otimes \varphi) \hat{\Delta}_{q}=\mathcal{F} \hat{\Delta} \varphi(\cdot) \mathcal{F}^{-1}$;

(ii) $(\hat{\varepsilon} \otimes \iota)(\mathcal{F})=(\iota \otimes \hat{\varepsilon})(\mathcal{F})=1$, where $\hat{\varepsilon}$ is the trivial representation of $G$;

(iii) $(\varphi \otimes \varphi)(\mathcal{R})=\mathcal{F}_{21} q^{t} \mathcal{F}^{-1}$;

(iv) the associator $\Phi=(\iota \otimes \hat{\Delta})\left(\mathcal{F}^{-1}\right)\left(1 \otimes \mathcal{F}^{-1}\right)(\mathcal{F} \otimes 1)(\hat{\Delta} \otimes \iota)(\mathcal{F})$ coincides with the Drinfeld associator $\Phi_{K Z}$.

If $\mathcal{F} \in \mathcal{U}(G \times G)$ is an element satisfying (i) for some isomorphism $\varphi: \mathcal{U}\left(G_{q}\right) \rightarrow \mathcal{U}(G)$ extending the identification of the centers, then we say that $\mathcal{F}$ is a twist. If in addition $\varphi$ is a $*$-homomorphism and $\mathcal{F}$ is unitary, we say that $\mathcal{F}$ is a unitary twist. If all four conditions (i)-(iv) are satisfied, we talk about Drinfeld twists and unitary Drinfeld twists.

Proof of Theorem 1.1. The existence of a Drinfeld twist in the formal deformation setting is due to Drinfeld [14, 15]. There it is proved by inductive cohomological arguments, so the twist makes sense only as a formal power series and a priori cannot be specialized to a complex deformation parameter. The result implies equivalence of certain braided tensor categories. A constructive proof of this equivalence was later given by Kazhdan and Lusztig [27, 28, the advantage being that the specialization makes sense (for nonzero complex parameters different from nontrivial roots of unity). It also implies the existence of a Drinfeld twist. The construction of Kazhdan and Lusztig was further clarified and extended by Etingof and Kazhdan [18, 19, 20].

Therefore there exist an isomorphism $\varphi: \mathcal{U}\left(G_{q}\right) \rightarrow \mathcal{U}(G)$ and a Drinfeld twist $\mathcal{F} \in \mathcal{U}(G \times G)$, and the only additional claim we make is that one can choose $\varphi$ to be $*$-preserving and $\mathcal{F}$ unitary.

Let us show first that $\varphi$ can be assumed to be $*$-preserving. Since every homomorphism of full matrix algebras is equivalent to a $*$-homomorphism, there exists an invertible element $u \in \mathcal{U}(G)$ such that the homomorphism

$$
\varphi_{u}=u \varphi(\cdot) u^{-1}
$$

is $*$-preserving. We may assume $\hat{\varepsilon}(u)=1$. Then

$$
\mathcal{F}_{u}=(u \otimes u) \mathcal{F} \hat{\Delta}\left(u^{-1}\right)
$$

is a Drinfeld twist for $\varphi_{u}$. Indeed, the conditions (i) and (ii) are obviously satisfied. To show (iii) recall that $t$ is $\mathfrak{g}$-invariant, i.e. $[t, \hat{\Delta}(x)]=0$ for any $x$. In particular, $\hat{\Delta}(u)$ commutes with $t$ and recalling that $\hat{\Delta}^{o p}=\hat{\Delta}$ we get

$$
\begin{aligned}
\left(\varphi_{u} \otimes \varphi_{u}\right)(\mathcal{R}) & =(u \otimes u) \mathcal{F}_{21} q^{t} \mathcal{F}^{-1}\left(u^{-1} \otimes u^{-1}\right) \\
& =(u \otimes u) \mathcal{F}_{21} \hat{\Delta}\left(u^{-1}\right) q^{t} \hat{\Delta}(u) \mathcal{F}^{-1}\left(u^{-1} \otimes u^{-1}\right)=\left(\mathcal{F}_{u}\right)_{21} q^{t} \mathcal{F}_{u}^{-1} .
\end{aligned}
$$


Finally, a direct computation shows that the new associator

$$
\Phi_{u}=(\iota \otimes \hat{\Delta})\left(\mathcal{F}_{u}^{-1}\right)\left(1 \otimes \mathcal{F}_{u}^{-1}\right)\left(\mathcal{F}_{u} \otimes 1\right)(\hat{\Delta} \otimes \iota)\left(\mathcal{F}_{u}\right)
$$

equals $\hat{\Delta}^{(2)}(u) \Phi \hat{\Delta}^{(2)}\left(u^{-1}\right)$. It remains to recall that $\Phi$ is $\mathfrak{g}$-invariant, since $\hat{\Delta}_{q}$ is coassociative. This is also clear by definition of $\Phi_{K Z}$, as $t$ is $\mathfrak{g}$-invariant and hence $\Phi\left(t_{12}, t_{23}\right)$ is $\mathfrak{g}$-invariant.

Assuming now that $\varphi: \mathcal{U}\left(G_{q}\right) \rightarrow \mathcal{U}(G)$ is a $*$-isomorphism and $\mathcal{E}$ a Drinfeld twist, we assert that the unitary $\mathcal{F}$ in the polar decomposition $\mathcal{E}=\mathcal{F}|\mathcal{E}|$ is a unitary Drinfeld twist for $\varphi$. Indeed, since $\hat{\Delta}_{q}, \hat{\Delta}$ and $\varphi$ are $*$-homomorphisms, condition (i) on $\mathcal{E}$ implies that

$$
\mathcal{E} \hat{\Delta} \varphi(\cdot) \mathcal{E}^{-1}=\left(\mathcal{E}^{-1}\right)^{*} \hat{\Delta} \varphi(\cdot) \mathcal{E}^{*},
$$

that is, $\mathcal{E}^{*} \mathcal{E}$ is $\mathfrak{g}$-invariant. It follows that $|\mathcal{E}|$ is also $\mathfrak{g}$-invariant. Hence

$$
\mathcal{E} \hat{\Delta} \varphi(\cdot) \mathcal{E}^{-1}=\mathcal{F}|\mathcal{E}| \hat{\Delta} \varphi(\cdot)|\mathcal{E}|^{-1} \mathcal{F}^{-1}=\mathcal{F} \hat{\Delta} \varphi(\cdot) \mathcal{F}^{-1},
$$

so condition (i) for $\mathcal{F}$ is satisfied. Condition (ii) is also obviously satisfied. Turning to (iii) recall that the $R$-matrix has the property $\mathcal{R}^{*}=\mathcal{R}_{21}$. So applying the $*$-operation and then the flip to the identity $(\varphi \otimes \varphi)(\mathcal{R})=\mathcal{E}_{21} q^{t} \mathcal{E}^{-1}$ we get $(\varphi \otimes \varphi)(\mathcal{R})=\left(\mathcal{E}^{-1}\right)_{21}^{*} q^{t} \mathcal{E}^{*}$. Therefore

$$
\left(\mathcal{E}^{*} \mathcal{E}\right)_{21} q^{t}=q^{t} \mathcal{E}^{*} \mathcal{E}
$$

and hence $|\mathcal{E}|_{21} q^{t}=q^{t}|\mathcal{E}|$. It follows that

$$
(\varphi \otimes \varphi)(\mathcal{R})=\mathcal{E}_{21} q^{t} \mathcal{E}^{-1}=\mathcal{F}_{21}|\mathcal{E}|_{21} q^{t}|\mathcal{E}|^{-1} \mathcal{F}^{-1}=\mathcal{F}_{21} q^{t} \mathcal{F}^{-1} .
$$

It remains to check (iv). Consider the new associator

$$
\Phi_{0}=(\iota \otimes \hat{\Delta})\left(\mathcal{F}^{-1}\right)\left(1 \otimes \mathcal{F}^{-1}\right)(\mathcal{F} \otimes 1)(\hat{\Delta} \otimes \iota)(\mathcal{F}) .
$$

We have to show that $\Phi_{0}=\Phi$. Since $|\mathcal{E}|$ is $\mathfrak{g}$-invariant, one easily checks that

$$
\Phi=(\iota \otimes \hat{\Delta})\left(|\mathcal{E}|^{-1}\right)\left(1 \otimes|\mathcal{E}|^{-1}\right) \Phi_{0}(|\mathcal{E}| \otimes 1)(\hat{\Delta} \otimes \iota)(|\mathcal{E}|) .
$$

Since $\Phi_{0}$ is defined by the unitary element $\mathcal{F}$, it is itself unitary. Since $\Phi=\Phi_{K Z}$ is also unitary, taking the inverses in the above identity and then applying the $*$-operation we get

$$
\Phi=(\iota \otimes \hat{\Delta})(|\mathcal{E}|)(1 \otimes|\mathcal{E}|) \Phi_{0}\left(|\mathcal{E}|^{-1} \otimes 1\right)(\hat{\Delta} \otimes \iota)\left(|\mathcal{E}|^{-1}\right) .
$$

Therefore

$(\iota \otimes \hat{\Delta})\left(|\mathcal{E}|^{-1}\right)\left(1 \otimes|\mathcal{E}|^{-1}\right) \Phi_{0}(|\mathcal{E}| \otimes 1)(\hat{\Delta} \otimes \iota)(|\mathcal{E}|)=(\iota \otimes \hat{\Delta})(|\mathcal{E}|)(1 \otimes|\mathcal{E}|) \Phi_{0}\left(|\mathcal{E}|^{-1} \otimes 1\right)(\hat{\Delta} \otimes \iota)\left(|\mathcal{E}|^{-1}\right)$.

Since $|\mathcal{E}|$ is $\mathfrak{g}$-invariant, the positive operators $(\iota \otimes \hat{\Delta})(|\mathcal{E}|)$ and $1 \otimes|\mathcal{E}|$, as well as $|\mathcal{E}| \otimes 1$ and $(\hat{\Delta} \otimes \iota)(|\mathcal{E}|)$, commute. So we can write

$$
\Phi_{0}((|\mathcal{E}| \otimes 1)(\hat{\Delta} \otimes \iota)(|\mathcal{E}|))^{2}=((1 \otimes|\mathcal{E}|)(\iota \otimes \hat{\Delta})(|\mathcal{E}|))^{2} \Phi_{0}
$$

Consequently

$$
\Phi_{0}(|\mathcal{E}| \otimes 1)(\hat{\Delta} \otimes \iota)(|\mathcal{E}|)=(1 \otimes|\mathcal{E}|)(\iota \otimes \hat{\Delta})(|\mathcal{E}|) \Phi_{0},
$$

and returning to (1.2) we get $\Phi=\Phi_{0}$.

Remark 1.2. For any $*$-isomorphism $\varphi: W^{*}\left(G_{q}\right) \rightarrow W^{*}(G)$ extending the identification of the centers, the existence of a unitary twist satisfying also condition (ii) follows immediately from the fact that the fusion rules for $G$ and $G_{q}$ are the same. Then one can modify the twist to satisfy condition (iii) using the symmetrization procedure of Drinfeld together with the identity

$$
\mathcal{R}^{*} \mathcal{R}=\hat{\Delta}_{q}\left(q^{C_{q}}\right)\left(q^{-C_{q}} \otimes q^{-C_{q}}\right),
$$

where $C_{q}=\varphi^{-1}(C)$ and $C=-\sum_{k} x_{k}^{2}$ is the Casimir, and the identity

$$
t=\frac{1}{2}(\hat{\Delta}(C)-1 \otimes C-C \otimes 1) .
$$


Note in passing that the above two identities imply that

$$
(\varphi \otimes \varphi)\left(\mathcal{R}^{*} \mathcal{R}\right)=\mathcal{F} q^{2 t} \mathcal{F}^{*}
$$

holds for any unitary twist $\mathcal{F}$.

Therefore the key condition is number (iv). In the formal deformation setting Drinfeld proves a stronger result, so it makes sense to ask the following question. Given a unitary twist $\mathcal{F}$ satisfying conditions (i)-(iii), does there exist a $\mathfrak{g}$-invariant unitary $\mathcal{E}$ such that $\mathcal{E}_{21}=\mathcal{E}$ and the associator defined by $\mathcal{F} \mathcal{E}$ coincides with the Drinfeld associator?

\section{DiRAC OPERATOR}

Recall that the Dirac operator $D$ on a spin manifold $M$ is defined as the composition

$$
\Gamma(S) \stackrel{\tilde{\nabla}}{\rightarrow} \Gamma\left(T^{*} M \otimes S\right) \stackrel{\sim}{\rightarrow} \Gamma(T M \otimes S) \stackrel{c}{\rightarrow} \Gamma(S)
$$

of the Clifford action $c$ on the spin bundle $S$ with the spin connection $\tilde{\nabla}$, using the metric to identify tangent and cotangent bundles. Thus with respect to an orthonormal local frame $\left\{e_{i}\right\}_{i}$ the Dirac operator is given by $D=\sum_{i} c\left(e_{i}\right) \tilde{\nabla}_{e_{i}}$.

Turning to the Dirac operator on $G$, trivialize the complexified tangent bundle $T G$ by left translations and identify $\Gamma(T G)$ with $C^{\infty}(G) \otimes \mathfrak{g}$. Define a Riemannian metric on $G$ using the form on $\mathfrak{g}$ introduced earlier multiplied by -1 . The Levi-Civita connection is given by

$$
\nabla_{f \otimes x}=f \partial(x) \otimes 1+\frac{1}{2} f \otimes \operatorname{ad}(x),
$$

where $\partial$ is the representation of $U \mathfrak{g}$ by left-invariant differential operators.

Denote by $\mathrm{Cl}(\mathfrak{g})$ the complex Clifford algebra of $\mathfrak{g}$ and by $\gamma: \mathfrak{g} \rightarrow \mathrm{Cl}(\mathfrak{g})$ the canonical embedding, so $\mathrm{Cl}(\mathfrak{g})$ is generated by $\gamma(x), x \in \mathfrak{g}$, and $\gamma(x)^{2}=(x, x) 1$. We regard $\mathrm{Cl}(\mathfrak{g})$ as a $*$-algebra by requiring the map $\gamma$ to be $*$-preserving. The spin group $\operatorname{Spin}(\mathfrak{g})$ is the connected Lie subgroup of the group of invertible elements of $\mathrm{Cl}(\mathfrak{g})$ with real Lie algebra spanned by the elements $\gamma\left(x_{k}\right) \gamma\left(x_{l}\right), k \neq l$. It acts on $\mathrm{Cl}(\mathfrak{g})$ by inner automorphisms. The adjoint action of $G$ on $\mathfrak{g}$ extends to an action of $G$ on $\mathrm{Cl}(\mathfrak{g})$ which lifts to a homomorphism $G \rightarrow \operatorname{Spin}(\mathfrak{g})$. At the Lie algebra level it is given by

$$
\mathfrak{g} \ni x \mapsto \widetilde{\operatorname{ad}}(x):=\frac{1}{4} \sum_{k} \gamma\left(x_{k}\right) \gamma\left(\left[x, x_{k}\right]\right) .
$$

We denote by the same symbol $\widetilde{a d}$ the corresponding homomorphism $\mathcal{U}(G) \rightarrow \mathrm{Cl}(\mathfrak{g})$. Note that by definition the map $\gamma$ is equivariant, so $\gamma([x, y])=\widetilde{\operatorname{ad}}(x), \gamma(y)]$ for $x, y \in \mathfrak{g}$.

Fix a spin module, that is, an irreducible *-representation $s: \mathrm{Cl}(\mathfrak{g}) \rightarrow B(\mathbb{S})$. Recall that if $\mathfrak{g}$ is even dimensional then $s$ is unique up to equivalence and faithful, and there are two possibilities for $s$ in the odd dimensional case. Identifying the smooth sections of the spin bundle $S=G \times \mathbb{S}$ with $C^{\infty}(G) \otimes \mathbb{S}$, the spin connection is

$$
\tilde{\nabla}_{f \otimes x}=f \partial(x) \otimes s(1)+\frac{1}{2} f \otimes s \widetilde{\operatorname{ad}}(x),
$$

The Clifford action of $1 \otimes x$ is given by $1 \otimes s \gamma(x)$. Hence using the orthonormal global frame $\left\{e_{k}=1 \otimes x_{k}\right\}_{k}$, we see that the Dirac operator $D: C^{\infty}(G) \otimes \mathbb{S} \rightarrow C^{\infty}(G) \otimes \mathbb{S}$ is given by

$$
D=\sum_{k}\left(\partial\left(x_{k}\right) \otimes s \gamma\left(x_{k}\right)+\frac{1}{2} \otimes s\left(\gamma\left(x_{k}\right) \widetilde{\operatorname{ad}}\left(x_{k}\right)\right)\right) .
$$

This can be written as $D=(\partial \otimes s)(\mathcal{D})$, where

$$
\mathcal{D}=\sum_{k}\left(x_{k} \otimes \gamma\left(x_{k}\right)+\frac{1}{2} \otimes \gamma\left(x_{k}\right) \widetilde{\operatorname{ad}}\left(x_{k}\right)\right)
$$


is an element of the non-commutative Weil algebra $U \mathfrak{g} \otimes \mathrm{Cl}(\mathfrak{g})$, see [1].

Remark 2.1. One can use other connections than the Levi-Civita one to define a Dirac operator by varying the coefficient $\frac{1}{2}$ in the above expressions [37]. Taking 0 one gets an operator corresponding to the reductive connection, and taking $\frac{1}{3}$ one gets Kostant's cubic Dirac operator [30].

Now fix a unitary twist $\mathcal{F}$ corresponding to a *-isomorphism $\varphi: W^{*}\left(G_{q}\right) \rightarrow W^{*}(G)$. Define the universal quantum Dirac operator $\mathcal{D}_{q} \in \mathcal{U}\left(G_{q}\right) \otimes \mathrm{Cl}(\mathfrak{g})$ by

$$
\mathcal{D}_{q}=\left(\varphi^{-1} \otimes \iota\right)\left((\iota \otimes \widetilde{\mathrm{ad}})(\mathcal{F}) \mathcal{D}(\iota \otimes \widetilde{\mathrm{ad}})\left(\mathcal{F}^{*}\right)\right) .
$$

Denote by $\mathbb{C}\left[G_{q}\right]$ the linear span of matrix coefficients of finite dimensional representations of $G_{q}$. It is a Hopf $*$-algebra with comultiplication $\Delta_{q}$, and $\mathcal{U}\left(G_{q}\right)$ is its dual space. Let $\left(L^{2}\left(G_{q}\right), \pi_{r, q}, \xi_{q}\right)$ be the GNS-triple defined by the Haar state on $\mathbb{C}\left[G_{q}\right]$. The left $\hat{\pi}_{r, q}$ and right $\partial_{q}$ regular representations of $W^{*}\left(G_{q}\right)$ on $L^{2}\left(G_{q}\right)$ are defined by

$$
\hat{\pi}_{r, q}(\omega) \pi_{r, q}(a) \xi_{q}=\left(\omega S^{-1} \otimes \pi_{r, q}\right) \Delta_{q}(a) \xi_{q},
$$

where $S$ is the antipode on $\mathbb{C}\left[G_{q}\right]$, and

$$
\partial_{q}(\omega) \pi_{r, q}(a) \xi_{q}=\left(\pi_{r, q} \otimes \omega\right) \Delta_{q}(a) \xi_{q}=a_{(1)}(\omega) \pi_{r, q}\left(a_{(0)}\right) \xi_{q} .
$$

Definition 2.2. The quantum Dirac operator $D_{q}$ is the unbounded operator on $L^{2}\left(G_{q}\right) \otimes \mathbb{S}$ defined by

$$
D_{q}=\left(\partial_{q} \otimes s\right)\left(\mathcal{D}_{q}\right) .
$$

Remark 2.3. The element $\mathcal{D}_{q}$ depends a priori on the choice of $\varphi$ and $\mathcal{F}$. Is it true that $\mathcal{D}$ commutes with all $\mathfrak{g}$-invariant elements in $(\iota \otimes \widetilde{\mathrm{ad}})(U \mathfrak{g} \otimes U \mathfrak{g})$ ? This is the case for $\mathrm{SU}(2)$, see Example 2.6 below, but this case is special since then $s \gamma$ coincides with $s \widetilde{\text { ad }}$ up to a scalar factor. If the answer is yes in general then $\mathcal{D}_{q}$ does not depend on $\mathcal{F}$ for fixed $\varphi$. On the other hand, the dependence on $\varphi$ is very mild. Namely, if we replace $\varphi$ by $\varphi^{\prime}$ then there exists a unitary $v \in W^{*}(G)$ such that $\varphi^{\prime}=v \varphi(\cdot) v^{*}$ and $\mathcal{F}_{v}=(v \otimes v) \mathcal{F} \hat{\Delta}\left(v^{*}\right)$ is a unitary twist for $\varphi^{\prime}$. Since $\mathcal{D}$ commutes with the image of $(\iota \otimes \widetilde{\mathrm{ad}}) \hat{\Delta}$, for the element $\mathcal{D}_{q}^{\prime}$ defined by $\varphi^{\prime}$ and $\mathcal{F}_{v}$ we get $\mathcal{D}_{q}^{\prime}=(1 \otimes \widetilde{\operatorname{ad}}(v)) \mathcal{D}_{q}\left(1 \otimes \widetilde{\operatorname{ad}}\left(v^{*}\right)\right)$.

The quantum group $G_{q}$ acts on itself from the left and from the right, and the operator $D_{q}$ is equivariant with respect to these two actions. More formally, we have two coactions of $\mathbb{C}\left[G_{q}\right]$ on itself. They can be implemented by the representations $\hat{\pi}_{r, q}(\cdot) \otimes 1$ and $\partial_{q} \times s \widetilde{\operatorname{ad}}_{q}=\left(\partial_{q} \otimes s \widetilde{\operatorname{ad}}_{q}\right) \hat{\Delta}_{q}$ of $W^{*}\left(G_{q}\right)$, where $\widetilde{\operatorname{ad}}_{q}=\widetilde{\operatorname{ad}} \varphi: W^{*}\left(G_{q}\right) \rightarrow \mathrm{Cl}(\mathfrak{g})$. Then we have the following.

Proposition 2.4. The universal quantum Dirac operator $\mathcal{D}_{q} \in \mathcal{U}\left(G_{q}\right) \otimes \mathrm{Cl}(\mathfrak{g})$ commutes with all elements of the form $\left(\iota \otimes \widetilde{\operatorname{ad}}_{q}\right) \hat{\Delta}_{q}(x)$, where $x \in W^{*}\left(G_{q}\right)$. In particular, the quantum Dirac operator $D_{q}$ commutes with all operators of the form $\hat{\pi}_{r, q}(x) \otimes 1$ and $\left(\partial_{q} \times s \widetilde{\operatorname{ad}}_{q}\right)(x)$.

Proof. Recall that as $t=-\sum_{k} x_{k} \otimes x_{k}$ is $\mathfrak{g}$-invariant and the map $\gamma$ is equivariant, the element $(\iota \otimes \gamma)(t)$ commutes with any element of the form $(\iota \otimes \widetilde{\mathrm{ad}}) \hat{\Delta}(x)$, and similarly $\sum_{k} \gamma\left(x_{k}\right) \widetilde{\operatorname{ad}}\left(x_{k}\right)$ commutes with any element in the image of ad. So $\mathcal{D}$ commutes with any element of the form $(\iota \otimes \widetilde{\mathrm{ad}}) \hat{\Delta}(x)$. Thus $(\varphi \otimes \iota)\left(\mathcal{D}_{q}\right)$ commutes with any element of the form

$$
(\iota \otimes \widetilde{\operatorname{ad}})\left(\mathcal{F} \hat{\Delta} \varphi(x) \mathcal{F}^{*}\right)=(\varphi \otimes \widetilde{\operatorname{ad}} \varphi) \hat{\Delta}_{q}(x)=\left(\varphi \otimes \widetilde{\operatorname{ad}}_{q}\right) \hat{\Delta}_{q}(x), \quad x \in \mathcal{U}\left(G_{q}\right),
$$

so $\mathcal{D}_{q}$ commutes with $\left(\iota \otimes \widetilde{\operatorname{ad}}_{q}\right) \hat{\Delta}_{q}(x)$.

By applying $\partial_{q} \otimes s$ we see that $D_{q}$ commutes with $\left(\partial_{q} \times s \widetilde{\operatorname{ad}}_{q}\right)(x)$. Finally $D_{q}$ commutes with $\hat{\pi}_{r, q}(x) \otimes 1$ simply because $\hat{\pi}_{r, q}(x)$ commutes with $\partial_{q}(y)$ for all $y$. 
Next note that by definition the operator $D_{q}$ is unitarily equivalent to $D$. In particular, $D_{q}$ is self-adjoint and its spectrum is the same as that of $D$. Recall that one can compute the squares of the eigenvalues of $D$ by using the Weitzenböck formula:

$$
\mathcal{D}^{2}=\frac{1}{2}(\iota \otimes \widetilde{\mathrm{ad}}) \hat{\Delta}(C)+\frac{1}{2} C \otimes 1+\frac{1}{4} \otimes \widetilde{\operatorname{ad}}(C) .
$$

Recall also that $\widetilde{\operatorname{ad}}(C)=3\|\rho\|^{2}$, where $\rho$ is half the sum of the positive roots, which can be seen using the well-known result of Kostant 29] that the representation $s \widetilde{\text { ad }}$ is equivalent to several copies of the irreducible representation with highest weight $\rho$, and that the image of $C$ under an irreducible representation with highest weight $\lambda$ is the scalar $\|\lambda+\rho\|^{2}-\|\rho\|^{2}$. Therefore

$$
\mathcal{D}^{2}=\frac{1}{2}(\iota \otimes \widetilde{\mathrm{ad}}) \hat{\Delta}(C)+\frac{1}{2} C \otimes 1+\frac{3}{4}\|\rho\|^{2} .
$$

For $\mathcal{D}_{q}$ this can be reformulated as follows.

Proposition 2.5. We have

It follows that

$$
\mathcal{D}_{q}^{2}=\frac{1}{2}\left(\iota \otimes \widetilde{\operatorname{ad}}_{q}\right) \hat{\Delta}_{q}\left(C_{q}\right)+\frac{1}{2} C_{q} \otimes 1+\frac{3}{4}\|\rho\|^{2} .
$$

$$
q^{2 \mathcal{D}_{q}^{2}}=\left(q^{2 C_{q}+\frac{9}{2}\|\rho\|^{2}} \otimes 1\right)\left(\iota \otimes \widetilde{\operatorname{ad}}_{q}\right)\left(\mathcal{R}^{*} \mathcal{R}\right) .
$$

Proof. The first identity follows immediately from definitions and the Weitzenböck formula. The second follows from (1.3) and the equality $\widetilde{\operatorname{ad}}_{q}\left(C_{q}\right)=3\|\rho\|^{2}$.

The proposition shows that $\mathcal{D}_{q}^{2}$ does not depend on the choice of $\mathcal{F}$. Moreover, one can get an explicit formula for $q^{2 \mathcal{D}_{q}^{2}}$ in terms of the generators of $U_{q} \mathfrak{g}$ (recall that $C_{q}$ can also be expressed in terms of the $R$-matrix by $q^{-2 C_{q}}=\hat{m}_{q}\left(\iota \otimes \hat{S}_{q}\right)\left(\mathcal{R}^{*} \mathcal{R}\right)$, where $\hat{m}_{q}$ and $\hat{S}_{q}$ are the multiplication and the antipode on $\mathcal{U}\left(G_{q}\right)$ ).

As in the classical case, the Weitzenböck formula allows one to compute the spectral subspaces of $D_{q}^{2}$. Namely, let $\tilde{V}_{\lambda, q} \subset L^{2}\left(G_{q}\right)$ be the linear span of the matrix coefficients of an irreducible representation with highest weight $\lambda$. Then $\tilde{V}_{\lambda, q} \otimes \mathbb{S}$ is $\left(\partial_{q} \times s \widetilde{\operatorname{ad}}_{q}\right)\left(W^{*}\left(G_{q}\right)\right)$-invariant, and if $V \subset$ $\tilde{V}_{\lambda, q} \otimes \mathbb{S}$ is an irreducible submodule with highest weight $\mu$, then $D_{q}^{2}$ acts on $V$ as the scalar

$$
\frac{1}{2}\|\mu+\rho\|^{2}+\frac{1}{2}\|\lambda+\rho\|^{2}-\frac{1}{4}\|\rho\|^{2} .
$$

It is worth recalling that if we use the reductive connection instead of the Levi-Civita one, then using a similar result we can compute the spectrum of $D_{q}$ completely, as then for any eigenvalue $\beta$ the number $-\beta$ is again an eigenvalue with the same multiplicity [21].

Example 2.6. Consider the simplest case $G=\mathrm{SU}(2)$. Then $\mathrm{Cl}(\mathfrak{g})$ can be identified with the algebra $B\left(V_{\frac{1}{2}}\right) \oplus B\left(V_{\frac{1}{2}}\right)$ in such a way that $\widetilde{\mathrm{ad}}=\pi_{\frac{1}{2}} \oplus \pi_{\frac{1}{2}}$ and $\gamma(x)=\sqrt{2}\left(\pi_{\frac{1}{2}}(x),-\pi_{\frac{1}{2}}(x)\right), x \in \mathfrak{g}$. Choose $s: B\left(V_{\frac{1}{2}}\right) \oplus B\left(V_{\frac{1}{2}}\right) \rightarrow B\left(V_{\frac{1}{2}}\right)$ to be the projection on the first factor. We then see that the map $(\sqrt{2})^{-1} s \gamma$ coincides with the restriction of $s \widetilde{\text { ad }}$ to $\mathfrak{g}$, in particular, it extends to a homomorphism $\mathcal{U}(G) \rightarrow B\left(V_{\frac{1}{2}}\right)$. This implies that the element $\mathcal{D}$ commutes with any element of the form $(\iota \otimes \widetilde{\mathrm{ad}})(\mathcal{E})$, where $\mathcal{E}$ is a $\mathfrak{g}$-invariant unitary, since $t$ commutes with any such unitary by virtue of (1.4). Hence for any fixed $\varphi$ the operator $\mathcal{D}_{q}$ is independent of the twist $\mathcal{F}$. Therefore by Remark 2.3 we conclude that $\mathcal{D}_{q}$ is unique up to the inner automorphism of $\mathcal{U}\left(G_{q}\right) \otimes \mathrm{Cl}(\mathfrak{g})$ defined by a unitary of the form $1 \otimes \widetilde{\operatorname{ad}}(u), u \in W^{*}\left(G_{q}\right)$.

Since in our case $\sum_{k} \widetilde{\operatorname{ad}}\left(x_{k}^{2}\right)=-\widetilde{\operatorname{ad}}(C)=-\frac{3}{2}$, we have

$$
(\iota \otimes s)(\mathcal{D})=-\sqrt{2}(\iota \otimes s \widetilde{\mathrm{ad}})(t)-\frac{3 \sqrt{2}}{4},
$$


whence

$$
q^{-\sqrt{2}(\iota \otimes s)(\mathcal{D})}=q^{\frac{3}{2}}(\iota \otimes s \widetilde{\mathrm{ad}})\left(q^{2 t}\right) .
$$

Recall that by (1.5) we have $(\varphi \otimes \varphi)\left(\mathcal{R}^{*} \mathcal{R}\right)=\mathcal{F} q^{2 t} \mathcal{F}^{*}$. It follows that

$$
q^{-\sqrt{2} D_{q}}=q^{\frac{3}{2}}\left(\partial_{q} \otimes s \widetilde{\operatorname{ad}}_{q}\right)\left(\mathcal{R}^{*} \mathcal{R}\right) .
$$

To get an explicit expression for $q^{-\sqrt{2} D_{q}}$, consider the standard generators $e=X_{1}, f=Y_{1}, k=K_{1}$ of $U_{q} \mathfrak{g}$. The representation $s \widetilde{\operatorname{ad}}_{q}$ is an irreducible representation of spin $\frac{1}{2}$, so with an appropriate choice of basis we have

$$
e \mapsto\left(\begin{array}{ll}
0 & 1 \\
0 & 0
\end{array}\right), \quad f \mapsto\left(\begin{array}{cc}
0 & 0 \\
1 & 0
\end{array}\right), \quad k \mapsto\left(\begin{array}{cc}
q^{\frac{1}{2}} & 0 \\
0 & q^{-\frac{1}{2}}
\end{array}\right) .
$$

Recall next that the $R$-matrix has the form

$$
\mathcal{R}=q^{2 \log _{q} k \otimes \log _{q} k} \sum_{n=0}^{\infty} R_{n}(q)(k e)^{n} \otimes\left(f k^{-1}\right)^{n},
$$

where $\log _{q}$ is the usual logarithm with base $q$ and $R_{0}(q)=1$ and $R_{1}(q)=q-q^{-1}$. Since $\left(s \widetilde{\operatorname{ad}}_{q}\right)\left(f^{n}\right)=$ 0 for $n \geq 2$, we get

$$
\left(\iota \otimes s \widetilde{\operatorname{ad}}_{q}\right)(\mathcal{R})=\left(\begin{array}{cc}
k & 0 \\
q^{-\frac{1}{2}}\left(q-q^{-1}\right) e & k^{-1}
\end{array}\right)
$$

whence

$$
q^{-\sqrt{2} D_{q}}=q^{\frac{3}{2}}\left(\begin{array}{cc}
\partial_{q}\left(k^{2}+q^{-1}\left(q-q^{-1}\right)^{2} f e\right) & q^{-\frac{1}{2}}\left(q-q^{-1}\right) \partial_{q}\left(f k^{-1}\right) \\
q^{-\frac{1}{2}}\left(q-q^{-1}\right) \partial_{q}\left(k^{-1} e\right) & \partial_{q}\left(k^{-2}\right)
\end{array}\right)
$$

\section{SpeCtral triple}

Our next goal is to study commutators of $D_{q}$ with regular functions on $G_{q}$.

Proposition 3.1. For any $a \in \mathbb{C}\left[G_{q}\right]$ we have

$$
\left[D_{q}, \pi_{r, q}(a) \otimes 1\right]=-\left(\pi_{r, q}\left(a_{(0)}\right) \otimes 1\right)\left(\partial_{q} \varphi^{-1} \otimes s\right)\left(a_{(1)} \varphi^{-1} \otimes \iota \otimes \iota\right)\left(U T U^{*}\right),
$$

where

$$
U=(\iota \otimes \iota \otimes \widetilde{\mathrm{ad}})((\mathcal{F} \otimes 1)(\hat{\Delta} \otimes \iota)(\mathcal{F}))
$$

is a unitary operator in $W^{*}(G) \bar{\otimes} W^{*}(G) \otimes \mathrm{Cl}(\mathfrak{g})$, the operator $T \in \mathcal{U}(G \times G) \otimes \mathrm{Cl}(\mathfrak{g})$ is defined by

$$
T=(\iota \otimes \iota \otimes \gamma)\left(t_{13}\right)+(\iota \otimes \iota \otimes \gamma)\left(t_{23}\right)-(\iota \otimes \iota \otimes \widetilde{\mathrm{ad}})\left(\Phi^{*}\right)(\iota \otimes \iota \otimes \gamma)\left(t_{23}\right)(\iota \otimes \iota \otimes \widetilde{\mathrm{ad}})(\Phi),
$$

and

$$
\Phi=(\iota \otimes \hat{\Delta})\left(\mathcal{F}^{*}\right)\left(1 \otimes \mathcal{F}^{*}\right)(\mathcal{F} \otimes 1)(\hat{\Delta} \otimes \iota)(\mathcal{F}) \in W^{*}(G) \bar{\otimes} W^{*}(G) \bar{\otimes} W^{*}(G)
$$

is the associator defined by the unitary twist $\mathcal{F}$.

Note that in the case $q=1$ we can take $\mathcal{F}=1$, then $U=1, \Phi=1, T=(\iota \otimes \iota \otimes \gamma)\left(t_{13}\right)=$ $-\sum_{k} x_{k} \otimes 1 \otimes \gamma\left(x_{k}\right)$, and we recover the familiar formula

$$
\left[D, \pi_{r}(a) \otimes 1\right]=\sum_{k} a_{(1)}\left(x_{k}\right) \pi_{r}\left(a_{(0)}\right) \otimes s \gamma\left(x_{k}\right)=c(d a) .
$$


Proof of Proposition 3.1. Since $\pi_{r, q}(a) \otimes 1$ commutes with $\sum_{k} 1 \otimes s\left(\gamma\left(x_{k}\right) \widetilde{\operatorname{ad}}\left(x_{k}\right)\right)$, it is only the part $-(\iota \otimes \gamma)(t)$ of $\mathcal{D}$ which contributes to the commutator. Thus the commutator is the difference of

$$
\left(\pi_{r, q}(a) \otimes 1\right)\left(\partial_{q} \varphi^{-1} \otimes s\right)\left((\iota \otimes \widetilde{\mathrm{ad}})(\mathcal{F})(\iota \otimes \gamma)(t)(\iota \otimes \widetilde{\mathrm{ad}})\left(\mathcal{F}^{*}\right)\right)
$$

and

$$
\left(\partial_{q} \varphi^{-1} \otimes s\right)\left((\iota \otimes \widetilde{\mathrm{ad}})(\mathcal{F})(\iota \otimes \gamma)(t)(\iota \otimes \widetilde{\mathrm{ad}})\left(\mathcal{F}^{*}\right)\right)\left(\pi_{r, q}(a) \otimes 1\right)
$$

Applying (3.2) to a vector $\pi_{r, q}(b) \xi_{q} \otimes \zeta$ with $b \in \mathbb{C}\left[G_{q}\right]$ and $\zeta \in \mathbb{S}$, by definition (2.1) of $\partial_{q}$ we get

$$
\left(a_{(1)} b_{(1)} \varphi^{-1} \otimes s\right)\left((\iota \otimes \widetilde{\mathrm{ad}})(\mathcal{F})(\iota \otimes \gamma)(t)(\iota \otimes \widetilde{\mathrm{ad}})\left(\mathcal{F}^{*}\right)\right)\left(\pi_{r, q}\left(a_{(0)} b_{(0)}\right) \xi_{q} \otimes \zeta\right)
$$

For any $c, d \in \mathbb{C}\left[G_{q}\right]$ we have

$$
\begin{aligned}
\left(c d \varphi^{-1}\right. & \otimes \iota)\left((\iota \otimes \widetilde{\mathrm{ad}})(\mathcal{F})(\iota \otimes \gamma)(t)(\iota \otimes \widetilde{\mathrm{ad}})\left(\mathcal{F}^{*}\right)\right) \\
& =(c \otimes d \otimes \iota)\left(\hat{\Delta}_{q} \varphi^{-1} \otimes \iota\right)\left((\iota \otimes \widetilde{\mathrm{ad}})(\mathcal{F})(\iota \otimes \gamma)(t)(\iota \otimes \widetilde{\mathrm{ad}})\left(\mathcal{F}^{*}\right)\right) \\
& =\left(c \varphi^{-1} \otimes d \varphi^{-1} \otimes \iota\right)\left((\mathcal{F} \otimes 1)(\hat{\Delta} \otimes \iota)\left((\iota \otimes \widetilde{\mathrm{ad}})(\mathcal{F})(\iota \otimes \gamma)(t)(\iota \otimes \widetilde{\mathrm{ad}})\left(\mathcal{F}^{*}\right)\right)\left(\mathcal{F}^{*} \otimes 1\right)\right) \\
& =\left(c \varphi^{-1} \otimes d \varphi^{-1} \otimes \iota\right)\left(U(\hat{\Delta} \otimes \gamma)(t) U^{*}\right) .
\end{aligned}
$$

Therefore (3.3) equals

$$
\begin{aligned}
\left(a_{(1)} \varphi^{-1} \otimes b_{(1)} \varphi^{-1} \otimes s\right) & \left(U(\hat{\Delta} \otimes \gamma)(t) U^{*}\right)\left(\pi_{r, q}\left(a_{(0)} b_{(0)}\right) \xi_{q} \otimes \zeta\right) \\
= & \left(\pi_{r, q}\left(a_{(0)}\right) \otimes 1\right)\left(a_{(1)} \varphi^{-1} \otimes \partial_{q} \varphi^{-1} \otimes s\right)\left(U(\hat{\Delta} \otimes \gamma)(t) U^{*}\right)\left(\pi_{r, q}(b) \xi_{q} \otimes \zeta\right) .
\end{aligned}
$$

In other words, (3.2) is equal to

$$
\left(\pi_{r, q}\left(a_{(0)}\right) \otimes 1\right)\left(\partial_{q} \varphi^{-1} \otimes s\right)\left(a_{(1)} \varphi^{-1} \otimes \iota \otimes \iota\right)\left(U(\hat{\Delta} \otimes \gamma)(t) U^{*}\right) .
$$

Consider now the operator (3.1). We can write it as

$\left(\pi_{r, q}\left(a_{(0)}\right) \otimes 1\right)\left(\partial_{q} \varphi^{-1} \otimes s\right)\left(a_{(1)} \varphi^{-1} \otimes \iota \otimes \iota\right)\left((\iota \otimes \iota \otimes \widetilde{\mathrm{ad}})(1 \otimes \mathcal{F})(\iota \otimes \iota \otimes \gamma)\left(t_{23}\right)(\iota \otimes \iota \otimes \widetilde{\mathrm{ad}})\left(1 \otimes \mathcal{F}^{*}\right)\right)$.

Since $(\iota \otimes \iota \otimes \gamma)\left(t_{23}\right)$ commutes with $(\iota \otimes \iota \otimes \widetilde{\mathrm{ad}})(\iota \otimes \hat{\Delta})(\mathcal{F})$, instead of conjugating $(\iota \otimes \iota \otimes \gamma)\left(t_{23}\right)$ by $(\iota \otimes \iota \otimes \widetilde{\mathrm{ad}})(1 \otimes \mathcal{F})$ in the above expression, we can conjugate it by

$$
(\iota \otimes \iota \otimes \widetilde{\mathrm{ad}})((1 \otimes \mathcal{F})(\iota \otimes \hat{\Delta})(\mathcal{F}))=U(\iota \otimes \iota \otimes \widetilde{\mathrm{ad}})\left(\Phi^{*}\right) .
$$

Thus (3.1) equals

$$
\left(\pi_{r, q}\left(a_{(0)}\right) \otimes 1\right)\left(\partial_{q} \varphi^{-1} \otimes s\right)\left(a_{(1)} \varphi^{-1} \otimes \iota \otimes \iota\right)\left(U(\iota \otimes \iota \otimes \widetilde{\mathrm{ad}})\left(\Phi^{*}\right)(\iota \otimes \iota \otimes \gamma)\left(t_{23}\right)(\iota \otimes \iota \otimes \widetilde{\mathrm{ad}})(\Phi) U^{*}\right) .
$$

To summarize, the commutator $\left[D_{q}, \pi_{r, q}(a) \otimes 1\right]$ is equal to the difference of (3.5) and (3.4). Since $(\hat{\Delta} \otimes \iota)(t)=t_{13}+t_{23}$, this is exactly what the proposition states.

Corollary 3.2. The commutator $\left[D_{q}, \pi_{r, q}(a) \otimes 1\right]$ is bounded for all $a \in \mathbb{C}\left[G_{q}\right]$ if and only if the commutator

$$
\left[(\pi \otimes \iota \otimes \gamma)\left(t_{23}\right),(\pi \otimes \iota \otimes \widetilde{\mathrm{ad}})(\Phi)\right]
$$

is bounded for any finite dimensional representation $\pi: G \rightarrow B\left(V_{\pi}\right)$.

One can equivalently formulate the above condition by saying that the operator

$$
[1 \otimes \mathcal{D},(\pi \otimes \iota \otimes \widetilde{\mathrm{ad}})(\Phi)]
$$

affiliated with $B\left(V_{\pi}\right) \otimes W^{*}(G) \otimes \mathrm{Cl}(\mathfrak{g})$ is bounded for any finite dimensional representation $\pi$ of $G$. 
Proof of Corollary [3.2. First observe that $\left[D_{q}, \pi_{r, q}(a) \otimes 1\right]$ is bounded for all $a \in \mathbb{C}\left[G_{q}\right]$ if and only if the operator

$$
\left(a \varphi^{-1} \otimes \iota \otimes s\right)\left(U T U^{*}\right) \in \mathcal{U}(G) \otimes B(\mathbb{S})
$$

is bounded for all $a \in \mathbb{C}\left[G_{q}\right]$. Indeed, it is clear that boundedness of such operators implies boundedness of the commutators. Conversely, assume that all the commutators are bounded, and write $1 \otimes a$ as a finite sum of elements of the form $(b \otimes 1) \Delta_{q}(c)$ with $b, c \in \mathbb{C}\left[G_{q}\right]$. Since $\pi_{r, q}(1)=1$ and

$$
\left(\pi_{r, q}\left(b c_{(0)}\right) \otimes 1\right)\left(\partial_{q} \varphi^{-1} \otimes s\right)\left(c_{(1)} \varphi^{-1} \otimes \iota \otimes \iota\right)\left(U T U^{*}\right)
$$

is bounded by assumption, we conclude that

$$
\left(a \varphi^{-1} \otimes \partial_{q} \varphi^{-1} \otimes s\right)\left(U T U^{*}\right)
$$

is bounded. Then $\left(a \varphi^{-1} \otimes \iota \otimes s\right)\left(U T U^{*}\right)$ is bounded as the representation $\partial_{q} \varphi^{-1}$ is faithful.

Next notice that when $a$ runs through all elements of $\mathbb{C}\left[G_{q}\right]$, the functionals $a \varphi^{-1}$ run through the linear span of matrix coefficients of all finite dimensional representations of $G$. So to say that $\left(a \varphi^{-1} \otimes \iota \otimes s\right)\left(U T U^{*}\right)$ is bounded for all $a \in \mathbb{C}\left[G_{q}\right]$ is the same as saying that

$$
(\pi \otimes \iota \otimes s)\left(U T U^{*}\right)
$$

is bounded for any finite dimensional unitary representation $\pi$ of $G$. Since $(\pi \otimes \iota \otimes s)(U)$ is unitary, this in turn is equivalent to boundedness of $(\pi \otimes \iota \otimes s)(T)$.

Now consider the expression for $(\pi \otimes \iota \otimes s)(T)$. The first term $(\pi \otimes \iota \otimes s \gamma)\left(t_{13}\right)$ is clearly bounded. On the other hand, since $(\pi \otimes \iota \otimes s \mathrm{ad})(\Phi)$ is unitary, the remaining part of $(\pi \otimes \iota \otimes s)(T)$ can be written as

$$
-(\pi \otimes \iota \otimes s \widetilde{\mathrm{ad}})\left(\Phi^{*}\right)\left[(\pi \otimes \iota \otimes s \gamma)\left(t_{23}\right),(\pi \otimes \iota \otimes s \widetilde{\mathrm{ad}})(\Phi)\right] .
$$

Therefore the commutators $\left[D_{q}, \pi_{r, q}(a) \otimes 1\right]$ are bounded if and only if

$$
(\iota \otimes \iota \otimes s)\left(\left[(\pi \otimes \iota \otimes \gamma)\left(t_{23}\right),(\pi \otimes \iota \otimes \widetilde{\mathrm{ad}})(\Phi)\right]\right)
$$

is bounded for any $\pi$. This is what we need if $\mathfrak{g}$ is even dimensional, as the representation $s$ is then faithful. In the odd dimensional case there exists another irreducible representation $\tilde{s}: \mathrm{Cl}(\mathfrak{g}) \rightarrow$ $B(\mathbb{S})$. Then $s \oplus \tilde{s}$ is faithful. The representations $s$ and $\tilde{s}$ are equivalent when restricted to the even subalgebra of $\mathrm{Cl}(\mathfrak{g})$. It follows that $s$ is isometric on the even subalgebra. But then we conclude that it is also isometric on the odd part of $\mathrm{Cl}(\mathfrak{g})$ by observing that $\|x\|^{2}=\left\|x^{*} x\right\|$ and if $x \in \mathrm{Cl}(\mathfrak{g})$ is odd then $x^{*} x$ is even. Now note that $(\iota \otimes \iota \otimes \gamma)\left(t_{23}\right)$ is odd, while $(\pi \otimes \iota \otimes \widetilde{\mathrm{ad}})(\Phi)$ is even, so their commutator is odd.

We now want to get an estimate of the norms of the above commutators in the case of the Drinfeld associator. But first we establish a couple of commutation relations.

Lemma 3.3. We have $[(\iota \otimes \gamma)(t),(\iota \otimes \widetilde{\mathrm{ad}})(t)]=0$.

Proof. By (1.4) we have

$$
2(\iota \otimes \widetilde{\mathrm{ad}})(t)=(\iota \otimes \widetilde{\mathrm{ad}}) \hat{\Delta}(C)-C \otimes 1-1 \otimes \widetilde{\mathrm{ad}}(C) .
$$

As we know, $(\iota \otimes \gamma)(t)$ commutes with any element of the form $(\iota \otimes \widetilde{\mathrm{ad}}) \hat{\Delta}(x)$. Thus it commutes with the first term on the right hand side of the above identity. It also clearly commutes with the second term. Finally, as we already remarked prior to Proposition 2.5, the third term is a scalar.

It is well-known and easy to check that $\left[t_{i k}, t_{i j}+t_{j k}\right]=0$ for nonequal $i, j, k$. In the spin representation we have a similar relation.

Lemma 3.4. We have $\left[(\iota \otimes \iota \otimes \gamma)\left(t_{13}\right),(\iota \otimes \iota \otimes \widetilde{\mathrm{ad}})\left(t_{12}+t_{23}\right)\right]=0$. 
Proof. Applying the flip to the first two factors, we can equivalently check

$$
\left[(\iota \otimes \iota \otimes \gamma)\left(t_{23}\right),(\iota \otimes \iota \otimes \widetilde{\mathrm{ad}})\left(t_{12}+t_{13}\right)\right]=0 .
$$

This follows immediately from $t_{12}+t_{13}=(\iota \otimes \hat{\Delta})(t)$.

The relations $\left[t_{i k}, t_{i j}+t_{j k}\right]=0$ imply consistency of the Knizhnik-Zamolodchikov equations, or equivalently, mutual commutativity of the Hamiltonians of the Gaudin model [16]. Similarly, using the two previous lemmas we get the following.

Lemma 3.5. For any $z \in \mathbb{C}$ we have

$$
\left[(\iota \otimes \iota \otimes \gamma)\left((1-z) t_{13}+t_{23}\right),(\iota \otimes \iota \otimes \widetilde{\mathrm{ad}})\left((z-1) t_{12}+z t_{23}\right)\right]=0 .
$$

Proof. By Lemma 3.4 we have

$$
(1-z)\left[(\iota \otimes \iota \otimes \gamma)\left(t_{13}\right),(\iota \otimes \iota \otimes \widetilde{\mathrm{ad}})\left((z-1) t_{12}+z t_{23}\right)\right]=(z-1)\left[(\iota \otimes \iota \otimes \gamma)\left(t_{13}\right),(\iota \otimes \iota \otimes \widetilde{\mathrm{ad}})\left(t_{12}\right)\right] .
$$

By Lemma 3.3 we also have

$$
\left[(\iota \otimes \iota \otimes \gamma)\left(t_{23}\right),(\iota \otimes \iota \otimes \widetilde{\mathrm{ad}})\left((z-1) t_{12}+z t_{23}\right)\right]=(z-1)\left[(\iota \otimes \iota \otimes \gamma)\left(t_{23}\right),(\iota \otimes \iota \otimes \widetilde{\mathrm{ad}})\left(t_{12}\right)\right] .
$$

So we just have to check that

$$
\left[(\iota \otimes \iota \otimes \gamma)\left(t_{13}+t_{23}\right),(\iota \otimes \iota \otimes \widetilde{\mathrm{ad}})\left(t_{12}\right)\right]=0 .
$$

This is indeed true as $t_{13}+t_{23}=(\hat{\Delta} \otimes \iota)(t)$.

We can now prove our main technical result.

Proposition 3.6. If $\Phi=\Phi_{K Z}$ is the Drinfeld associator, then for any finite dimensional unitary representation $\pi: G \rightarrow B\left(V_{\pi}\right)$ we have

$$
\left\|\left[(\pi \otimes \iota \otimes \gamma)\left(t_{23}\right),(\pi \otimes \iota \otimes \widetilde{\mathrm{ad}})(\Phi)\right]\right\| \leq 6\|(\pi \otimes \gamma)(t)\| .
$$

Proof. Fix finite dimensional unitary representations $\pi$ and $\pi^{\prime}$ of $G$. Put

$$
A=\left(\pi \otimes \pi^{\prime} \otimes \widetilde{\mathrm{ad}}\right)\left(t_{12}\right) \text { and } B=\left(\pi \otimes \pi^{\prime} \otimes \widetilde{\mathrm{ad}}\right)\left(t_{23}\right) .
$$

According to (1.1) we have

$$
\left(\pi \otimes \pi^{\prime} \otimes \widetilde{\mathrm{ad}}\right)(\Phi)=\lim _{a \rightarrow 0^{+}} a^{-\hbar B} G_{a}(1-a) a^{\hbar A},
$$

where $G_{a}$ is such that $G_{a}(a)=1$ and

$$
G_{a}^{\prime}(x)=\hbar\left(\frac{A}{x}+\frac{B}{x-1}\right) G_{a}(x) .
$$

Since all three operators $a^{-\hbar B}, G_{a}(1-a)$ and $a^{\hbar A}$ are unitary, to prove the proposition is suffices to show that

for all $a, x \in(0,1)$.

$$
\begin{gathered}
{\left[\left(\pi \otimes \pi^{\prime} \otimes \gamma\right)\left(t_{23}\right), a^{-\hbar B}\right]=0,} \\
\left\|\left[\left(\pi \otimes \pi^{\prime} \otimes \gamma\right)\left(t_{23}\right), G_{a}(x)\right]\right\| \leq 4\|(\pi \otimes \gamma)(t)\|, \\
\left\|\left[\left(\pi \otimes \pi^{\prime} \otimes \gamma\right)\left(t_{23}\right), a^{\hbar A}\right]\right\| \leq 2\|(\pi \otimes \gamma)(t)\|
\end{gathered}
$$

Equality (3.6) follows from Lemma 3.3.

To show (3.8) recall that $A$ commutes with

$$
\left(\pi \otimes \pi^{\prime} \otimes \gamma\right)\left(t_{13}+t_{23}\right)=\left(\pi \otimes \pi^{\prime} \otimes \gamma\right)(\hat{\Delta} \otimes \iota)(t) .
$$

Hence the left hand side of (3.8) equals $\left\|\left[\left(\pi \otimes \pi^{\prime} \otimes \gamma\right)\left(t_{13}\right), a^{\hbar A}\right]\right\|$. Since $a^{\hbar A}$ is unitary, the latter norm is not larger than

$$
2\left\|\left(\pi \otimes \pi^{\prime} \otimes \gamma\right)\left(t_{13}\right)\right\|=2\|(\pi \otimes \gamma)(t)\| .
$$


Turning to (3.7), for a fixed $a \in(0,1)$ consider the commutator

$$
L(x)=\left[\left(\pi \otimes \pi^{\prime} \otimes \gamma\right)\left((1-x) t_{13}+t_{23}\right), G_{a}(x)\right] .
$$

Then, as $G_{a}(x)$ is unitary and so $\left\|\left[\left(\pi \otimes \pi^{\prime} \otimes \gamma\right)\left(t_{13}\right), G_{a}(x)\right]\right\| \leq 2\|(\pi \otimes \gamma)(t)\|$, it is enough to check that

$$
\|L(x)\| \leq 2\|(\pi \otimes \gamma)(t)\|
$$

for all $x \in(0,1)$. We have for the derivative of $L$ that

$$
L^{\prime}(x)=\left[\left(\pi \otimes \pi^{\prime} \otimes \gamma\right)\left((1-x) t_{13}+t_{23}\right), \hbar\left(\frac{A}{x}+\frac{B}{x-1}\right) G_{a}(x)\right]-\left[\left(\pi \otimes \pi^{\prime} \otimes \gamma\right)\left(t_{13}\right), G_{a}(x)\right] .
$$

Since $\left(\pi \otimes \pi^{\prime} \otimes \gamma\right)\left((1-x) t_{13}+t_{23}\right)$ commutes with $\frac{A}{x}+\frac{B}{x-1}$ by Lemma 3.5, we thus see that $L$ satisfies the differential equation

$$
L^{\prime}(x)=\hbar\left(\frac{A}{x}+\frac{B}{x-1}\right) L(x)-\left[\left(\pi \otimes \pi^{\prime} \otimes \gamma\right)\left(t_{13}\right), G_{a}(x)\right]
$$

with initial condition $L(a)=0$. Consequently

$$
L(x)=-\int_{a}^{x} G_{y}(x)\left[\left(\pi \otimes \pi^{\prime} \otimes \gamma\right)\left(t_{13}\right), G_{a}(y)\right] d y,
$$

from which we get (3.9) using again unitarity of $G_{y}(x)$ and $G_{a}(y)$.

Therefore we get an equivariant spectral triple $\left(\mathbb{C}\left[G_{q}\right], L^{2}\left(G_{q}\right) \otimes \mathbb{S}, D_{q}\right)$. Since $D_{q}$ is unitarily equivalent to $D$, this spectral triple has the same summability properties as the classical one.

Recall next that if $\mathfrak{g}$ is of even dimension $2 m$ then the classical spectral triple is graded by the chirality element $\chi=i^{m} \gamma\left(x_{1}\right) \ldots \gamma\left(x_{2 m}\right)$. Since $1 \otimes \chi$ anticommutes with $\mathcal{D}$ and commutes with elements in the image of $\iota \otimes \widetilde{\mathrm{ad}}$, we see that $1 \otimes \chi$ anticommutes with $\mathcal{D}_{q}$, so our spectral triple for $G_{q}$ is even.

To summarize, we have the following result.

Theorem 3.7. If the operator $D_{q}$ is defined using a unitary Drinfeld twist then

$$
\left(\mathbb{C}\left[G_{q}\right], L^{2}\left(G_{q}\right) \otimes \mathbb{S}, D_{q}\right)
$$

is an equivariant spectral triple of the same parity as the dimension of $G$.

Remark 3.8.

(i) According to Remark 2.1 we can use connections $\nabla_{f \otimes x}=f \partial(x) \otimes 1+\lambda f \otimes \operatorname{ad}(x), \lambda \in \mathbb{R}$, to define Dirac operators. By the same procedure as before we then get Dirac operators on $G_{q}$. Since the commutators of these operators with $\pi_{r, q}(a) \otimes 1, a \in \mathbb{C}\left[G_{q}\right]$, do not depend on $\lambda$, the above theorem remains true for all such operators.

(ii) The Dirac operator on $G$ is closely related to Dirac operators on homogeneous spaces. Consider a homogeneous space $G / K$. Fix a spin module $\mathbb{S}_{\mathfrak{k}}$ for $\mathrm{Cl}(\mathfrak{k})$. Denote by $D_{\mathfrak{k}}$ the Dirac operator on the spin bundle over $G / K$ twisted by the bundle induced by the representation $K \rightarrow B\left(\mathbb{S}_{\mathfrak{k}}\right)$. On the other hand, we can can consider the restriction of the Dirac operator on $G$ to the space of $K$-invariant sections. These two operators can be expressed in terms of each other. The relation is most transparent when $G / K$ is even dimensional; then the spaces on which these operators act can be identified, and the difference of the operators is bounded.

Therefore using the Dirac operator $D_{q}$ on $G_{q}$ we obtain spectral triples on quantum homogeneous spaces. These are deformations of the above twisted Dirac operators (whenever they exist). In particular, if $K=T$ is the maximal torus we obtain a spectral triple on the quantum full flag manifold $G_{q} / T$ that is a deformation of the direct sum of $2^{[\mathrm{rank} \mathfrak{g} / 2]}$ copies of the spectral triple on $G / T$. 


\section{REFERENCES}

[1] A. Alekseev, E. Meinrenken, The non-commutative Weil algebra, Invent. Math. 139 (2000), 135-172.

[2] R. Bautista, A. Criscuolo, M. Durdević, M. Rosenbaum, J. D. Vergara, Quantum Clifford algebras from spinor representations, J. Math. Phys. 37 (1996), 5747-5775.

[3] P. S. Chakraborty, A. Pal, Equivariant spectral triples on the quantum SU(2) group, K-Theory 28 (2003), $107-126$.

[4] P. S. Chakraborty, A. Pal, On equivariant Dirac operators for $S U_{q}(2)$, preprint math.QA/0501019.

[5] P. S. Chakraborty, A. Pal, Equivariant spectral triples for $S U_{q}(\ell+1)$ and the odd dimensional quantum spheres, math.QA/0503689

[6] V. Chari, A. Pressley, A guide to quantum groups, Cambridge University Press, Cambridge, 1995.

[7] A. Connes, Noncommutative geometry, Academic Press, Inc., San Diego, CA, 1994.

[8] A. Connes, Cyclic cohomology, quantum group symmetries and the local index formula for $\mathrm{SU}_{q}(2)$, J. Inst. Math. Jussieu 3 (2004), 17-68.

[9] A. Connes, G. Landi, Noncommutative manifolds, the instanton algebra and isospectral deformations, Comm. Math. Phys. 221 (2001), 141-159.

[10] F. D'Andrea, L. Da̧browski, G. Landi, E. Wagner, Dirac operators on all Podles quantum spheres, preprint math.QA/0606480

[11] F. D'Andrea, L. Dạbrowski, G. Landi, The Isospectral Dirac Operator on the 4-dimensional Quantum Euclidean Sphere, preprint math.QA/0611100

[12] L. Dạbrowski, G. Landi, A. Sitarz, W. van Suijlekom, J. C. Várilly, The Dirac operator on $\mathrm{SU}_{q}(2)$, Comm. Math. Phys. 259 (2005), 729-759.

[13] L. Dą̧browski, A. Sitarz, Dirac operator on the standard Podleś quantum sphere, in: Noncommutative geometry and quantum groups (Warsaw, 2001), 49-58, Banach Center Publ., 61, Polish Acad. Sci., Warsaw, 2003.

[14] V. G. Drinfeld, Quasi-Hopf algebras, Leningrad Math. J. 1 (1990), 1419-1457.

[15] V. G. Drinfeld, On quasitriangular quasi-Hopf algebras and on a group that is closely connected with $\mathrm{Gal}(\overline{\mathbb{Q}} / \mathbb{Q})$, Leningrad Math. J. 2 (1991), 829-860.

[16] M. Gaudin, Diagonalisation d'une classe d'Hamiltoniens de spin, J. Physique 37 (1976), 1089-1098.

[17] P. I. Etingof, I. B. Frenkel, A. A. Kirillov Jr., Lectures on representation theory and Knizhnik-Zamolodchikov equations, Mathematical Surveys and Monographs, 58, American Mathematical Society, Providence, RI, 1998.

[18] P. Etingof, D. Kazhdan, Quantization of Lie bialgebras. I, Selecta Math. (N.S.) 2 (1996), 1-41.

[19] P. Etingof, D. Kazhdan, Quantization of Lie bialgebras. II, Selecta Math. (N.S.) 4 (1998), 213-231.

[20] P. Etingof, D. Kazhdan, Quantization of Lie bialgebras. VI, preprint math.QA/0004042.

[21] H. Fegan, The spectrum of the Dirac operator on a simply connected compact Lie group, Simon Stevin 61 (1987), 97-108.

[22] G. Fiore, Deforming maps for Lie group covariant creation and annihilation operators, J. Math. Phys. 39 (1998), 3437-3452.

[23] G. Fiore, Braided chains of q-deformed Heisenberg algebras, J. Phys. A 31 (1998), 5289-5298.

[24] G. Fiore, Drinfel'd twist and q-deforming maps for Lie group covariant Heisenberg algebrae, Rev. Math. Phys. 12 (2000), 327-359.

[25] I. Heckenberger, Spin geometry on quantum groups via covariant differential calculi, Adv. Math. 175 (2003), 197-242.

[26] C. Kassel, Quantum groups, Graduate Texts in Mathematics, 155, Springer-Verlag, New York, 1995.

[27] D. Kazhdan, G. Lusztig, Tensor structures arising from affine Lie algebras. III, J. Amer. Math. Soc. 7 (1994), $335-381$.

[28] D. Kazhdan, G. Lusztig, Tensor structures arising from affine Lie algebras. IV, J. Amer. Math. Soc. 7 (1994), $383-453$.

[29] B. Kostant, Clifford algebra analogue of the Hopf-Koszul-Samelson theorem, the $\rho$-decomposition $C(\mathfrak{g})=$ End $V_{\rho} \otimes$ $C(P)$, and the $\mathfrak{g}$-module structure of $\bigwedge \mathfrak{g}$, Adv. Math. 125 (1997), 275-350.

[30] B. Kostant, A cubic Dirac operator and the emergence of Euler number multiplets of representations for equal rank subgroups, Duke Math. J. 100 (1999), 447-501.

[31] U. Krähmer, Dirac operators on quantum flag manifolds, Lett. Math. Phys. 67 (2004), 49-59.

[32] S. Majid, Noncommutative Riemannian and spin geometry of the standard q-sphere, Comm. Math. Phys. 256 (2005), 255-285.

[33] S. Neshveyev, L. Tuset, A local index formula for the quantum sphere, Comm. Math. Phys. 254 (2005), 323-341.

[34] R. Owczarek, Dirac operator on the Podleś sphere, in: Clifford algebras and their applications (Ixtapa, 1999), Internat. J. Theoret. Phys. 40 (2001), 163-170.

[35] K. Schmüdgen, Commutator representations of covariant differential calculi, in: Noncommutative geometry and quantum groups (Warsaw, 2001), 189-198, Banach Center Publ., 61, Polish Acad. Sci., Warsaw, 2003.

[36] K. Schmüdgen, E. Wagner, Dirac operator and a twisted cyclic cocycle on the standard Podleś quantum sphere, J. Reine Angew. Math. 574 (2004), 219-235. 
[37] S. Slebarski, Dirac operators on a compact Lie group, Bull. London Math. Soc. 17 (1985), 579-583.

Department of Mathematics, University of Oslo, P.O. Box 1053 Blindern, N-0316 Oslo, Norway.

E-mail address: sergeyn@math.uio.no

Faculty of Engineering, Oslo University College, Cort Adelers St. 30, N-0254 Oslo, Norway.

E-mail address: Lars.Tuset@iu.hio.no 